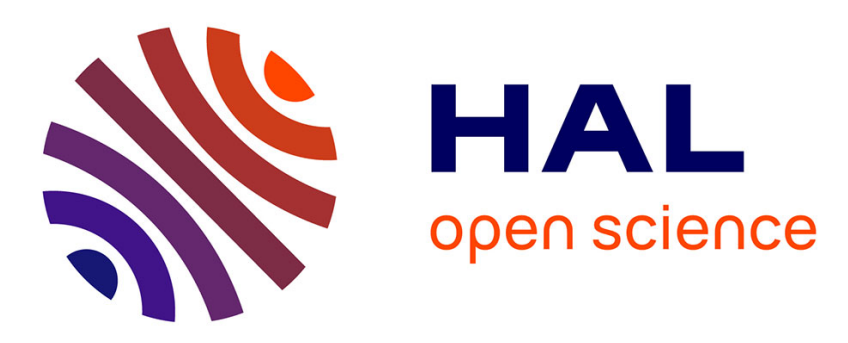

\title{
Markov chains in a Dirichlet Environment and hypergeometric integrals
}

Christophe Sabot

\section{To cite this version:}

Christophe Sabot. Markov chains in a Dirichlet Environment and hypergeometric integrals. Comptes rendus de l'Académie des sciences. Série I, Mathématique, 2002, 335 (11), pp.941-946. 10.1016/S1631073X(02)02580-3 . hal-00011151

\section{HAL Id: hal-00011151 https://hal.science/hal-00011151}

Submitted on 11 Oct 2005

HAL is a multi-disciplinary open access archive for the deposit and dissemination of scientific research documents, whether they are published or not. The documents may come from teaching and research institutions in France or abroad, or from public or private research centers.
L'archive ouverte pluridisciplinaire HAL, est destinée au dépôt et à la diffusion de documents scientifiques de niveau recherche, publiés ou non, émanant des établissements d'enseignement et de recherche français ou étrangers, des laboratoires publics ou privés. 


\title{
Markov chains in a Dirichlet Environment and hypergeometric integrals
}

\section{Chaînes de Markov en environnement de Dirichlet et intégrales hypergéométriques}

\author{
Christophe Sabot ${ }^{\mathrm{a}}$ \\ ${ }^{a} C N R S$, UMPA, ENS Lyon, 46 allée d'Italie, 69007 Lyon
}

\begin{abstract}
The aim of this text is to establish some relations between Markov chains in Dirichlet Environments on directed graphs and certain hypergeometric integrals associated with a particular arrangement of hyperplanes. We deduce from these relations and the computation of the connexion obtained by moving one hyperplane of the arrangement some new relations on important functionals of the Markov chain.

\section{Résumé}

Le but de ce texte est d'établir une relation entre les chaînes de Markov en environnement de Dirichlet sur des graphes orientés, et certaines intégrales hypergéométriques associées à un arrangement d'hyperplans. Nous déduisons du calcul de la connexion obtenue en bougeant un hyperplan des relations nouvelles sur des fonctionnelles importantes de ces marches.
\end{abstract}

Version française abrégée Les lois de Dirichlet jouent un role spécifique dans le contexte des marches aléatoires en environnement aléatoire, car la loi moyennée d'une marche aléatoire en environnement de Dirichlet est la loi d'une marche renforcée sur les arêtes orientées ( $\mathrm{cf}[1])$. De façon plus profonde, le but de cette note est d'établir un lien entre certaines fonctionnelles importantes de ces marches, étroitement liées à la transformée de Laplace du temps d'occupation des arêtes, et des intégrales hypergéométriques associées à un arrangement d'hyperplans particulier. On calcule alors la connexion obtenue en faisant

\footnotetext{
1 Je tiens à remercier N. Enriquez à qui cette note doit beaucoup.

Email address: csabot@umpa.ens-lyon.fr (Christophe Sabot).
} 
bouger un hyperplan; elle induit un système différentiel satisfait par les fonctionnelles de notre marche aléatoire. Nous pensons que les propriétés algébriques de cette connexion pourraient donner beaucoup d'information sur les marches aléatoires qui nous intéressent.

On se place dans le cadre général suivant : on considère un graphe orienté $G=(V, E)$ contenant un sommet cimetière $\delta$, dont aucune arête ne sort. On note $U=V \backslash\{\delta\}$, et on suppose qu'il existe un chemin simple orienté entre tout point $x$ de $U$ et $\delta$. On suppose aussi qu'on a un point $x_{0}$ dans $U$, à partir duquel on peut atteindre tout point de $V$ par un chemin orienté. On note $\bar{e}$ et $\underline{e}$ la tête et la queue d'une arête $e$. On se donne des poids positifs $\alpha_{e}$ sur chacune des arêtes $e$. On construit alors la chaîne de Markov en environnement aléatoire sur le graphe $G$ de la façon suivante : en chaque point $x$ de $U$ on tire des probabilités de sortie $\left(p_{e}\right)_{\underline{e}}=x$, indépendamment suivant une loi de Dirichlet de paramêtre $\left(\alpha_{e}\right)_{\underline{e}}=x$. On note $\mathbb{E}^{(\alpha)}$ la moyenne associée sur les environnements, et $P^{\left(p_{e}\right)}$ la loi de la chaîne de Markov sur le graphe $G$, partant de $x_{0}$ et arrêtée en $\delta$, obtenue à partir des probabilités de transition $\left(p_{e}\right)$. On note alors $G^{p}$ la fonction de Green de la marche tuée en $\delta$, de telle sorte que $z_{e}=G^{p}\left(x_{0}, \underline{e}\right) p_{e}$ est le nombre moyen de traversées de l'arête $e$ avant d'atteindre $\delta$. Le but de cette note est d'exprimer certaines fonctionnelles comme $\mathbb{E}^{(\alpha)}\left[e^{-<\lambda, z>} p_{T}\right]$, où $\left(\lambda_{e}\right) \in\left(\mathbb{R}_{+}^{*}\right)^{E}$ et $p_{T}$ est la probabilité de l'arbre couvrant $T$ orienté vers $\delta$ (pour la mesure de probabilité naturellement associé à la chaîne de Markov $P^{\left(p_{e}\right)}$ ), en fonction d'intégrales hypergéométriques associées à un arrangement d'hyperplan particulier. Cette correspondance est obtenue en faisant le changement de variables $\left(p_{e}\right) \mapsto\left(z_{e}\right)$.

Décrivons maintenant brièvement l'arrangement considéré. On note div $: \mathbb{R}^{E} \rightarrow \mathbb{R}^{U}$ l'opérateur div $(\theta)(x)=$ $\sum_{\underline{e}=x} \theta_{e}-\sum_{\bar{e}=x} \theta_{e}$ et $\mathcal{H}^{G, x_{0}}$ l'espace affine $\mathcal{H}^{G, x_{0}}=\left\{\left(z_{e}\right), \operatorname{div}(z)=\delta_{x_{0}}\right\}$. Les hyperplans $H_{e}=\left\{z_{e}=\right.$ $0\}$ forment un arrangement d'hyperplans $\mathcal{A}^{G, x_{0}}=\left(\mathcal{H}^{G, x_{0}},\left(H_{e}\right)_{e \in E}\right)$. Nous sommes particulierement intéressés par le domaine $\Delta=\left\{z_{e}>0\right\}$. Les bases de cet arrangement sont les familles $\left(H_{e_{1}}, \ldots, H_{|E|-|U|}\right)$ où $\left(e_{1}, \ldots, e_{|E|-|U|}\right)$ forment le complémentaire d'un arbre couvrant $T$ de $E$. On note alors $\omega_{T}=$ $\frac{d z_{e_{1}}}{z_{e_{1}}} \wedge \cdots \wedge \frac{d z_{e_{|E|-|U|}}}{z_{e_{|E|-|U|}}}$, et nous considérons les intégrales hypergéométriques $I_{\Delta, T}^{(\alpha)}(\lambda)$ définies en $(1)$. Les intégrales de ce type ont été intensivement étudiées (cf [2] pour un texte de présentation). En particulier, en bougeant un hyperplan ces intégrales satisfont un système différentiel ([2]), que nous calculons dans ce cas précis, cf théorème 3.1. Nous relions ces intégrales (en fait les intégrales obtenues à partir d'un graphe bipartite $\hat{G}$ simplement construit à partir de $G$ ) à la transformée de Laplace de la densité d'occupation des arêtes $z_{e}$, pondérée par le poids des arbres couvrants du graphe, cf théorème 2.1.

\section{Introduction}

Among random environments, Dirichlet environments play a special role since the annealed law of a random walk in a Dirichlet environment corresponds to the law of a reinforced random walk, on oriented edges (cf [1]). More deeply, the aim of this text is to show that random walks in Dirichlet environment have strong relations with certain hypergeometric integrals, which have been intensively studied (cf e.g. [2] for a review text). As a consequence of these relations, we are able to write a differential system satisfied by the Laplace transform of certain important functionals of the walks (this differential system is related to the Gauss-Manin connection of the arrangement, cf [2], chap 8, 10). We hope this new direction will be useful to understand the properties of Random Walks in Dirichlet Environment. The complete proofs of the results announced in this note will appear soon.

Let us now describe the model of Markov chains in random Dirichlet environment on directed graphs. Let $G=(V, E)$ be a finite directed graph, $V$ is the set of vertices and $E \subset V \times V$ is the set of edges. We denote by $\underline{e}$ (resp. $\bar{e})$ the origin (resp. the destination) of the edge $e$, so that $e=(\underline{e}, \bar{e})$. For simplicity we assume that there is no loop (i.e. edge of the type $(x, x))$. We suppose that the set of vertices can be 
decomposed in $V=U \sqcup\{\delta\}$, and that a base point $x_{0} \in U$ is given, such that: there is no edge with origin $\delta$; for all $x \in U$ there is a directed simple path from $x$ to $\delta$; for any $x$ in $U$, there is a directed simple path from $x_{0}$ to $x$, with the following simple definitions.

Definition 1.1 i) A simple path from vertices $x$ to $y, x \neq y$, is a set of edges $\left\{e_{1}, \cdots, e_{k}\right\}$ such that there is a list of distinct vertices $\left(x_{0}=x, x_{1}, \ldots, x_{k}=y\right)$ such that for all $j, 1 \leq j \leq k$, we have either $e_{j}=\left(x_{j-1}, x_{j}\right)$ or $e_{j}=\left(x_{j}, x_{j-1}\right)$. The path is directed if for all $j, e_{j}=\left(x_{j-1}, x_{j}\right)$.

ii) A (simple) cycle is a set of edges $\left\{e_{1}, \ldots, e_{k}\right\}$ such that there is a list of distinct vertices $\left(x_{0}, \ldots, x_{k-1}\right)$ such that for all $j, 1 \leq j \leq k$, we have either $e_{j}=\left(x_{j-1}, x_{j}\right)$ or $e_{j}=\left(x_{j}, x_{j-1}\right)$, with the convention that $x_{k}=x_{0}$. The cycle is directed if for all $j, e_{j}=\left(x_{j-1}, x_{j}\right)$.

iii) A spanning tree is a subset $T$ of edges which contains no cycle and which contains a simple path between any two vertices $x$ and $y$. The spanning tree $T$ is directed (towards $\delta$ ) if for any vertex $x$ in $U$ it contains a unique edge $e$ with origin $x$. In this case for any vertex $x$ the (simple) path from $x$ to $\delta$ in $T$ is directed.

Now, we construct some Markov chain on $G$ killed at $\{\delta\}$. We define the set of environments

$$
\tilde{\Delta}=\left\{\left(p_{e}\right) \in(0,1]^{E}, \quad \forall x \in U, \sum_{e, \underline{e}=x} p_{e}=1\right\}
$$

An element $\left(p_{e}\right)$ of $\tilde{\Delta}$ defines a Markov chain on $V$, starting at $x_{0}$, stopped at $\delta$, and with exit probabilities $\left(p_{e}\right)_{\underline{e}}=x$ at any point $x$ in $U$.

Let us now consider a set of positive weights $\left(\alpha_{e}\right)_{e \in E}$. For all $x$ in $U$ we set $\beta_{x}=\sum_{\underline{e}=x} \alpha_{e}$. We endow the set of environments $\tilde{\Delta}$ with the probability $\mu^{(\alpha)}$ with density

$$
\frac{\prod_{x \in U} \Gamma\left(\beta_{x}\right)}{\prod_{e \in E} \Gamma\left(\alpha_{e}\right)}\left(\prod_{e \in E} p_{e}^{\alpha_{e}-1}\right) d \lambda_{\tilde{\Delta}},
$$

where $d \lambda_{\tilde{\Delta}}$ is the measure on $\tilde{\Delta}$ given by $d \lambda_{\tilde{\Delta}}=\prod_{e \in \tilde{E}} d p_{e}$, where $\tilde{E}$ is obtained from $E$ by removing arbitrarily, for each vertex $x$, one edge with origin $x$ (one can easily see that $d \lambda_{\tilde{\Delta}}$ is independent of this choice). This means that the transition probabilities are chosen independently at each vertex $x$ under a Dirichlet law of parameter $\left(\alpha_{e}\right)_{\underline{e}}=x$.

We denote by $G_{U}^{p}$ the Green function of the Markov chain with transition probabilities $\left(p_{e}\right)$ killed at $\delta$, i.e.

$$
G_{U}^{p}(x, y)=E_{x}^{\left(p_{e}\right)}\left[\sum_{k=0}^{T_{\delta}-1} \mathbb{1}_{X_{k}=y}\right]=\left(I-P_{U}\right)_{x, y}^{-1},
$$

where $T_{\delta}$ is the first hitting time of $\delta$, and $P_{U}$ is the transition matrix of the Markov chain restricted to $U \times U$. For all edge $e \in E, z_{e}=G_{U}^{p}\left(x_{0}, \underline{e}\right) p_{e}$ is equal to the expected number of crossings of the edge $e$ before the killing time $T_{\delta}$. These values give of course considerable information on the behavior of the random walk $P_{x_{0}}^{\left(p_{e}\right)}$.

\section{The arrangement $\left(\mathcal{H}^{G, x_{0}},\left(H_{e}\right)_{e \in E}\right)$, and the change of variables.}

We suppose that we have a graph $G=(V, E)$ and a base point $x_{0}$ as in section 1 , and some (not necessarily positive) weights $\left(\alpha_{e}\right)_{e \in E}$. We define the divergence operator div $: \mathbb{R}^{E} \rightarrow \mathbb{R}^{U}$ by

$$
\operatorname{div}(\theta)(x)=\sum_{\underline{e}=x} \theta_{e}-\sum_{\bar{e}=x} \theta_{e}, \quad\left(\theta_{e}\right) \in \mathbb{R}^{E}, x \in U
$$


and $\mathcal{H}^{G, x_{0}}$ the affine space by

$$
\mathcal{H}^{G, x_{0}}=\left\{\left(z_{e}\right) \in \mathbb{R}^{E}, \quad \operatorname{div}(z)=\delta_{x_{0}}\right\},
$$

where $\delta_{x_{0}}$ is the Dirac mass at $x_{0}$. The hyperplanes $H_{e}=\left\{z_{e}=0\right\} \cap \mathcal{H}^{G, x_{0}}$ define an arrangement of hyperplanes in the affine space $\mathcal{H}^{G, x_{0}}$ that we denote

$$
\mathcal{A}^{G, x_{0}}=\left(\mathcal{H}^{G, x_{0}},\left(H_{e}\right)_{e \in E}\right) .
$$

The complement $\mathcal{H}^{G, x_{0}} \backslash \cup_{e} H_{e}$ determines some connected components, and we will be specially interested in a particular connected component $\Delta=\left\{\left(z_{e}\right) \in \mathcal{H}^{G, x_{0}}, z_{e}>0, \forall e \in E\right\}$. The crucial remark is that the function $\left(z_{e}\right)=\left(G_{U}^{p}\left(x_{0}, \underline{e}\right) p_{e}\right)$ takes its values in $\Delta$ for all environment $\left(p_{e}\right) \in \tilde{\Delta}$, thanks to the third assumption on $G, x_{0}$. In particular $\Delta$ is not empty. We recall that a basis of an arrangement is a maximal free subfamily of $\left(H_{e}\right)$.

Proposition 2.1 The bases of the arrangement $\mathcal{A}^{G, x_{0}}$ are exactly the subsets $\left\{H_{e}\right\}_{e \in T^{c}}$, for all spanning trees $T$.

We suppose given on $\mathcal{H}^{G, x_{0}}$ an arbitrary orientation. To any spanning tree $T$, we associate the differential form, with logarithmic poles

$$
\omega_{T}=\frac{d z_{e_{1}}}{z_{e_{1}}} \wedge \cdots \wedge \frac{d z_{e_{|E|-|U|}}}{z_{e_{|E|-|U|}}}
$$

where $\left\{e_{1}, \ldots, e_{|E|-|U|}\right\}=T^{c}$ and is ordered so that $\omega_{T}$ is positively oriented.

We will be interested in the following integrals (when they are well-defined): for all $\left(\lambda_{e}\right) \in(\mathbb{C})^{E}$, $\Re\left(\lambda_{e}\right)>0$ we set

$$
I_{\Delta, T}^{(\alpha)}(\lambda)=\int_{\Delta} e^{-<\lambda, z>} \prod_{e} z_{e}^{\alpha_{e}} \omega_{T}
$$

We want to relate some functional of the Markov chain defined in section 1 with hypergeometric integrals of the type (1). The Markov chain in Dirichlet environment defined on $G$ as in section (1) is not naturally related to the hypergeometric integral (1) for the graph $G$, but for a closely related graph $\hat{G}$.

The strategy is to construct a bipartite graph $\hat{G}=(\hat{V}, \hat{E})$ from the graph $G=(V, E)$ by duplication of the vertices of $U$. We define $\hat{V}=\hat{U} \cup\{\delta\}$, where $\hat{U}=\{-,+\} \times U$. To simplify notations, we denote $(-, x)$ (resp. $(+, x)$ ) by $x_{-}$(resp. $x_{+}$). We construct the set of edges $\hat{E}$ as follows: to any edge $e \in E$ we associate an edge $\hat{e} \in \hat{E}$ by $\hat{e}=\left(x_{+}, y_{-}\right)$if $e=(x, y)$ and $y \neq \delta$, and $\hat{e}=\left(x_{+}, \delta\right)$ if $y=\delta$. To any vertex $x$ in $U$ we associate an edge $\hat{e}_{x}=\left(x_{-}, x_{+}\right)$. We define the set of edges of $\hat{G}$ by $\hat{E}=\{\hat{e}, e \in E\} \cup\left\{\hat{e}_{x}, x \in U\right\}$.

Then, we define the affine space $\hat{\mathcal{H}}^{G, x_{0}}=\mathcal{H}^{\hat{G},\left(-, x_{0}\right)}=\left\{\left(\hat{z}_{e}\right) \in \mathbb{R}^{\hat{E}}, \quad \operatorname{div}\left(\left(\hat{z}_{e}\right)\right)=\delta_{\left(-, x_{0}\right)}\right\}$. It is clear that the affine spaces $\mathcal{H}^{G, x_{0}}$ and $\hat{\mathcal{H}}^{G, x_{0}}$ are isomorphic. Thus we always write $\mathcal{H}^{G, x_{0}}$ for $\hat{\mathcal{H}}^{G, x_{0}}$, and we set $z_{\hat{e}_{x}}=\sum_{\underline{e}=x} z_{e}$ for all vertex $x \in U$. The arrangement associated with the graph $\hat{G}$ is then $\hat{\mathcal{A}}^{G, x_{0}}=$ $\left(\mathcal{H}^{G, x_{0}},\left(H_{e}\right)_{e \in \hat{E}}\right)$. Compared with the arrangement associated with $G$, we see that they have the same underlying affine space $\mathcal{H}^{G, x_{0}}$, but the arrangement of $\hat{G}$ is richer since it contains all the hyperplanes of the arrangement of $G$ plus the hyperplanes $H_{\hat{e}_{x}}=\left\{z_{\hat{e}_{x}}=0\right\}=\left\{\sum_{e=x} z_{e}=0\right\}$, for all vertex $x$ in $U$.

Comparing the spanning trees, it is clear that any spanning tree $T^{T}$ of $G$ can be extended into a spanning tree $\hat{T}$ of $\hat{G}$ by adding the edges $\left\{e_{x}, x \in U\right\}$. Moreover, if $\hat{T}$ is a directed spanning tree of $\hat{G}$, then all the edges $\hat{e}_{x}$ are contained in $\hat{T}$ (cf definition of section 2), thus removing the edges $\left\{\hat{e}_{x}, x \in U\right\}$ we get a directed spanning tree of $G$. Thus, the directed spanning trees of $G$ and $\hat{G}$ are the same.

We give now the following weights to the edges of $\hat{E}: \alpha_{\hat{e}}=\alpha_{e}, e \in E, \alpha_{\hat{e}_{x}}=-\beta_{x}$. It is clear that the domain $\Delta$ of the arrangement $\mathcal{A}^{G, x_{0}}$ is also a domain of the arrangement $\hat{\mathcal{A}}^{G, x_{0}}$ since $z_{\hat{e}_{x}}=\sum_{\underline{e}=x} z_{e}$ is positive if all the $z_{e}$ are positive. For all $\lambda \in \mathbb{C}^{\hat{E}}, \Re\left(\lambda_{e}\right)>0$, and all spanning tree $\hat{T}$ of $\hat{G}$, we denote by 


$$
\hat{I}_{\hat{T}, \Delta}^{(\alpha)}(\lambda)=\int_{\Delta} e^{-<\lambda, z>}\left(\prod_{e \in \hat{E}} z_{e}^{\alpha_{e}}\right) \omega_{T} .
$$

the integral (1) for the graph $\hat{G}$ and the weights $\left(\alpha_{e}, e \in \hat{E}\right)$ such defined. Without loss of generality we can consider a function $\lambda$ which vanishes on the edges $\hat{e}_{x}$ (indeed, since $\hat{z}_{\hat{e}_{x}}=\sum_{\underline{e}=x} \hat{z}_{\hat{e}}$ ), so we may consider $\hat{I}_{\hat{T}, \Delta}(\lambda)$ as a function on $\mathbb{C}^{E}$.

Theorem 2.2 For all directed spanning tree $T$ of $G$, and for all $\lambda \in \mathbb{C}^{E}, \Re\left(\lambda_{e}\right)>0$, we have the following equality (and the integral are well-defined):

$$
C_{\alpha} \hat{I}_{\Delta, \hat{T}}^{(\alpha)}(\lambda)=\mathbb{E}^{(\alpha)}\left[e^{-<\lambda, z>}\left(\frac{\prod_{e \in T} p_{e}}{\operatorname{det}\left(I-P_{U}\right)}\right)\right],
$$

where on the left-hand side $C_{\alpha}=\prod_{x} \Gamma\left(\beta_{x}\right) / \prod_{e} \Gamma\left(\alpha_{e}\right)$ and $\hat{T}$ is the associated directed spanning tree in $\hat{G}$, and on the right-hand side $z_{e}=G^{p}\left(x_{0}, \underline{e}\right) p_{e}$ is the expected number of visits of the edge e by the Markov chain on $G$ starting at $x_{0}$ with transition probabilities $\left(p_{e}\right)$.

Sketch of the proof: We make the (not so easy) change of variables $i: \tilde{\Delta} \rightarrow \Delta$, given by $\left(p_{e}\right) \mapsto z_{e}=$ $G_{U}^{p}\left(x_{0}, \underline{e}\right) p_{e}$.

This last formula has an important probabilistic meaning. Indeed, we see that we get the Laplace transform of the occupation density of the edge, which is an important probabilistic quantity, weighted by the probability of a directed spanning tree (for the natural probability measure associated with $\left(p_{e}\right)$ ). This law on spanning trees contains a lot of information on the initial Markov chain. For example, by the Wilson algorithm (cf [3]), the law of the unique (directed) simple path from $x_{0}$ to $\delta$ is the law of the loop erased random walk from $x$ to $\delta$, obtained from the Markov chain $P^{\left(p_{e}\right)}$. Moreover, summing on all directed spanning trees we exactly get the Laplace transform of the occupation density of the edges.

\section{The Gauss-Manin connection}

We consider a graph $G$ and some weights $\alpha_{e}$ such that the integrals $I_{\Delta, T}(\lambda)$ are well-defined (actually, we are mainly interested in the graph $\hat{G}$ obtained from a graph $G$ with positive weights, as described in section 2). We denote by $\mathcal{T}$ the set of spanning trees of $G$. To any cycle $C$ of $G$ (given with an arbitrary orientation) we associate a linear form $l_{C}$ on $\mathbb{R}^{E}$, and to any simple path $\sigma$ from $x_{0}$ to $\delta$ we associate a linear form $l_{\sigma}$, given by

$$
l_{C}(\lambda)=\sum_{e \in C} \epsilon_{C}(e) \lambda_{e}, \quad l_{\sigma}(\lambda)=\sum_{e \in \sigma} \epsilon_{\sigma}(e) \lambda_{e}, \quad\left(\lambda_{e}\right) \in \mathbb{R}^{E},
$$

where $\epsilon_{C}(e)$ is equal to +1 (resp. - 1 ) if $e$ is directed according to (resp. in opposition to) the orientation of $C$, and $\epsilon_{\sigma}(e)$ is equal to 1 (or -1 ) if $e$ is directed from $x_{0}$ to $\delta$ (or from $\delta$ to $x_{0}$ ) in the path $\sigma$. We define the manifold $M=\mathbb{C}^{E} \backslash \cup_{C}$ cycle $\operatorname{ker} l_{C}$, where the logarithmic differential forms $\frac{d l_{C}}{l_{C}}$ are well-defined.

Let $\left(f_{T}\right)_{T \in \mathcal{T}}$ be the canonical base of $\mathbb{R}^{\mathcal{T}}$. To all simple cycle $C$ of $G$, we associate the endomorphism $\Omega_{C}$ of $\mathbb{R}^{\mathcal{T}}$, given by

$$
\Omega_{C}\left(f_{T}\right)=\sum_{e \in C} \epsilon_{C}\left(e_{0}, e\right) \alpha_{e} f_{T \cup\left\{e_{0}\right\} \backslash\{e\}},
$$

if there is an edge $e_{0}$ in $T^{c}$ such that $C \subset T \cup\left\{e_{0}\right\}$ and $\Omega_{C}\left(f_{T}\right)=0$ otherwise (in the last formula, $\epsilon_{C}\left(e_{0}, e\right)$ is equal either to +1 , resp. -1 , if the directions of $e$ and $e^{\prime}$ are the same, resp. opposite, in the cycle $C$ ). To any simple path $\sigma$ from $x_{0}$ to $\delta$, we associate the diagonal endomorphism $\Omega_{\sigma}$ of $\mathbb{R}^{\mathcal{T}}$, with coefficients $\left(\Omega_{\sigma}\right)_{T, T}=1$ if $\sigma \subset T$, and 0 otherwise. It is clear that $\Omega_{\sigma}$ is a projector on the subspace generated by 
the spanning trees containing $\sigma$. Concerning the matrices $\Omega_{C}$ we easily get $\left(\Omega_{C}\right)^{2}=\left(\sum_{e \in C} \alpha_{e}\right) \Omega_{C}$, which means that $\frac{\Omega_{C}}{\sum_{e \in C} \alpha_{e}}$ is a projector.

Theorem 3.1 The vector $I_{\Delta}(\lambda)=\left(I_{\Delta, T}(\lambda)\right)_{T \in \mathcal{T}}$ satisfies $d I_{\Delta}(\lambda)=-\Omega I_{\Delta}(\lambda)$, on $M \cap\left\{\Re\left(\lambda_{e}\right)>0\right\}$, where $d$ is the differential operator in the variables $(\lambda)$, and $\Omega$ is the $\mathcal{T} \times \mathcal{T}$ matrix (with differential form coefficients), given by

$$
\Omega=\sum_{\sigma} d l_{\sigma}(\lambda) \Omega_{\sigma}+\sum_{C} \frac{d l_{C}(\lambda)}{l_{C}(\lambda)} \Omega_{C}
$$

where the first sum is taken over all simple paths from $x_{0}$ to $\delta$ and the second summation is taken over all simple cycles of $G$.

Proof: If $T$ is a spanning tree, and $e$ an edge in $T^{c}$, there is a unique cycle $C_{T}^{e}$ contained in $T \cup\{e\}$, that we orient by convention according to the direction of $e$. Moreover, there is a unique simple path $\sigma_{T}$ from $x_{0}$ to $\delta$ in $T$, that we orient from $x_{0}$ to $\delta$. The proof is based on two types of relation. The first one is of cohomological nature: let $e_{0}$ be in $T^{c}$ then

$$
l_{C_{T}^{e_{0}}}(\lambda) \int_{\Delta} z_{e_{0}} e^{-<\lambda, z>}\left(\prod_{e \in E} z_{e}^{\alpha_{e}}\right) \omega_{T}=\left(\sum_{e^{\prime} \in C_{T}^{e_{0}}} \epsilon_{C_{T}^{e_{0}}}\left(e^{\prime}\right) I_{\Delta, T \cup\left\{e_{0}\right\} \backslash\left\{e^{\prime}\right\}}(\lambda)\right) .
$$

The second relation comes from the condition $\operatorname{div}(z)=\delta_{x_{0}}$, which implies that for all $\left(\lambda_{e}\right) \in \mathbb{R}^{E}$ and for all spanning tree $T:\langle z, \lambda\rangle=l_{\sigma_{T}}(\lambda)+\sum_{e \in T^{c}} z_{e} l_{C_{T}^{e}}(\lambda)$.

The operator $\nabla=d+\Omega$ defines a connection on the vector bundle $M \times \mathbb{R}^{\mathcal{T}}$, and theorem 3.1 says that $I_{\Delta}(\lambda)$ is a flat section of this connection. This connection is actually integrable, and we describe the structure equations. Let us introduce a definition. We define the genus of a subset $S \subset E$ as the size of the maximal free family of cycles contained in $S$ (we say that a family $\left(C_{1}, \ldots, C_{k}\right)$ of cycles is free if the associated functions $\left(\chi_{C_{1}}, \ldots, \chi_{C_{k}}\right)$ are free, where $\left.\chi_{C}=\sum_{C} \epsilon^{C}(e) \delta_{e}\right)$.

Proposition 3.1 The matrices $\Omega_{C}$ and $\Omega_{\sigma}$ satisfy the following commutation relations:

i) $\left[\Omega_{C}, \Omega_{C^{\prime}}\right]=0$ if $C$ and $C^{\prime}$ are disjoint or if the genus of $C \cup C^{\prime}$ is not 2 .

ii) $\left[\Omega_{\sigma}, \Omega_{\sigma^{\prime}}\right]=0$ for all $\sigma, \sigma^{\prime}$.

iii) $\left[\Omega_{C}, \Omega_{\sigma}\right]=0$ if $C$ and $\sigma$ are disjoint or the genus of $\sigma \cup C$ is not 1 .

iv) If $S \subset C$ has genus 2 , then either it contains 2 disjoint cycles, or exactly 3 cycles. In the last case, these 3 cycles $C_{1}, C_{2}, C_{3}$ satisfy the following commutation relation $\left[\Omega_{C_{1}}+\Omega_{C_{2}}+\Omega_{C_{3}}, \Omega_{C_{i}}\right]=0$, for all $i=1,2,3$.

v) If $\sigma_{1}$ and $\sigma_{2}$ are two simple paths from $x_{0}$ to $\delta$ such that the genus of $\sigma_{1} \cup \sigma_{2}$ is 1 , i.e. such that $\sigma_{1} \cup \sigma_{2}$ contains a unique cycle $C$, then $\left[\Omega_{\sigma_{1}}+\Omega_{\sigma_{2}}, \Omega_{C}\right]=0$.

These relations imply that the connection $\nabla$ is integrable, i.e. that $\nabla^{2}=\Omega \wedge \Omega=0$.

Sketch of the proof: the commutation relations i), ii), iii), iv), v) imply that $\Omega \wedge \Omega=0$, once we remark that $\frac{d l_{C_{1}}}{l_{C_{1}}} \wedge \frac{d l_{C_{2}}}{l_{C_{2}}}-\frac{d l_{C_{1}}}{l_{C_{1}}} \wedge \frac{d l_{C_{3}}}{l_{C_{3}}}+\frac{d l_{C_{2}}}{l_{C_{2}}} \wedge \frac{d l_{C_{3}}}{l_{C_{3}}}=0$, for $C_{1}, C_{2}, C_{3}$ in the configuration iv), and that $d l_{\sigma_{1}} \wedge \frac{d l_{C}}{l_{C}}=$ $d l_{\sigma_{2}} \wedge \frac{d l_{C}}{l_{C}}$ in the configuration $\left.\mathrm{v}\right)$. The commutation relations are obtained by direct computation.

\section{References}

[1] Enriquez, Nathanaël; Sabot, Christophe, Edge oriented reinforced random walks and RWRE. C. R. Math. Acad. Sci. Paris 335 (2002), no. 11, 941-946.

[2] Orlik, Peter; Terao, Hiroaki, Arrangements and hypergeometric integrals. MSJ Memoirs, 9. Mathematical Society of Japan, Tokyo, 2001. 
[3] Propp, J. G. and Wilson, D. B., How to get a perfectly random sample from a generic Markov chain and generate a random spanning tree of a directed graph. J. Algorithms 27, (1998), 170-217. 